\title{
Atmospheric Pressure Plasma Jet Treatment of Human Hair Fibers
}

\author{
Cansu Öngel • Merve Keleş • Erhan Acar • \\ Özgür Birer
}

Received: 30 September 2014/Revised: 27 November 2014/ Accepted: 15 January 2015/Published online: 4 February 2015

(c) Springer International Publishing AG 2015

\begin{abstract}
Human hair fibers in virgin and dyed forms were treated with atmospheric pressure helium, helium/ oxygen, argon, and argon/oxygen plasma jets at $20 \mathrm{~W}$ of power. The effects of 10-min plasma treatment on surface morphology and chemistry were studied by scanning electron microscopy and X-ray photoelectron spectroscopy, respectively. The plasma treatment was quite effective for removing the organic residues from the surface and creating oxidized functional groups. Helium plasma had a mild cleaning effect on the surfaces while argon/oxygen plasma had the strongest corrosive effect. Mild hydrogen peroxide treatment for the same duration had neither the cleaning nor the oxidizing power of the plasma jets. These types of plasma jets have the potential to replace peroxide treatment. The corrosive jets can be used to restore dyed hair fibers. In addition, the jets can be used to clean the surfaces of hair fibers to prepare samples for analytical investigations where the organic residues may induce problems.
\end{abstract}

C. Öngel · Ö. Birer ( $₫)$

Department of Chemistry, Koç University, Rumeli Feneri Yolu, Sariyer, 34450 Istanbul, Turkey

e-mail: obirer@ku.edu.tr

\section{Keleş}

Department of Molecular Biology and Genetics, Koç University, Rumeli Feneri Yolu, Sariyer, 34450 Istanbul, Turkey

\section{E. Acar . Ö. Birer}

Materials Science and Engineering Graduate Program, Koç University, Rumeli Feneri Yolu, Sariyer, 34450 Istanbul, Turkey

Ö. Birer

KUYTAM Surface Science and Technology Center, Koç University, Rumeli Feneri Yolu, Sariyer, 34450 Istanbul, Turkey
Keywords Atmospheric pressure plasma jets - Hair treatment $\cdot$ Surface cleaning $\cdot$ Plasma corrosion

\section{Introduction}

Atmospheric pressure cold plasma jets have been used in textile, plastics, and automotive industry for a while [1-6]. Such plasmas are not under thermal equilibrium; typical ion temperatures are about $300-400 \mathrm{~K}$ while the electron temperatures are much higher [4]. This feature enables the users to treat sensitive surfaces without the risk of thermal damage. Atmospheric pressure plasma jets can meet the practical requirements for surface activation, cleaning, decontamination, or etching by changing the gas mixture and/or excitation source [1-5].

Despite many laboratories employing atmospheric pressure plasmas, even the reports on treatment of the same surfaces may not always agree. The reason for the multitude of information is that cold plasmas can be excited with pulsed DC, AC, RF, and microwave sources thus generating a spread of electron and ion densities and energies with various designs [7-9]. Furthermore, the presence of highly energetic electrons, (deep) UV radiation, active radicals, and metastable atoms in the plasma leads to competing complex reaction mechanisms.

Natural and synthetic textile fibers have been treated with atmospheric pressure plasma systems to alter wettability, surface roughness, chemical composition, and dye uptake $[6,10-15]$. We could find only one report about the air plasma treatment of hair fibers to alter surface chemistry [16]. Unfortunately, the details of the plasma application, high-resolution microscopy images, or detailed surface compositions were not presented. The current work reports the detailed results of atmospheric pressure cold plasma jet 
treatment of human hair fibers, to our knowledge, for the first time. Homemade plasma jets were used to treat the surface of human hair fibers and the surface chemistry and morphology changes were investigated.

The human hair is made out of three sections: the outermost cuticle, the middle cortex, and the innermost medulla [17]. The medulla is a thin cylindrical section rich in lipids, while the cysteine-rich cortex surrounds the medulla. The outermost cuticle is made out of overlapping segments typically 8-11 layers. Each cell of the cuticle has a top ca. 3-nm layer called the epicuticle. The chemical composition of the top layer has been determined in detail by various analytical techniques [18, 19]. The epicuticle normally has a hydrophobic surface due to the extensive coverage by 18-methyl eicosanoic acid (18-MEA) (ca. $25 \%$ ) containing lipids as well as the proteins (ca. $75 \%$ ) rich in cross-linked cysteine. The 18-MEA layer is chemically bonded to the amorphous keratin through thioester or ester groups [20, 21]. However, 18-MEA layer is described to have a heterogeneous coverage [22-24].

The top layer can be stripped off via processes such as peroxide treatment [25-27], or ultraviolet exposure [26]. During the chemical process, i.e., bleaching, the chemistry of the top lipid layer and, when exposed, underlying proteins are altered [19] resulting in the changes in surface energy and hydrophobicity [24, 25], luster [28], and mechanical properties [21]. The surface of a bleached hair fiber has lower cysteine and higher cysteic acid $\left(-\mathrm{SO}_{3}{ }^{-}\right)$ contents due to the removal of the 18-MEA layer and oxidation of cysteine groups [19]. However, for cosmetic applications, it is desirable to remove the top lipid layer to decorate the surface with negatively charged cysteic acid groups since they can interact with polar and reactive groups of coloring agents more efficiently. On the other hand, since the permanent coloring products contain copious amounts of alkaline peroxide in an oxidative environment, the hair fiber must also be protected by adding fatty alcohols, cationic surfactants, or polymers to the dying emulsions [27]. Therefore, a search for milder reaction conditions is always active, especially for those with intolerance and possible dermatological reactions (2-15\% of general population) to bleaching agents [27].

In this work, atmospheric pressure plasma jets are evaluated for their potential to strip the lipid layers from the top epicuticle layer. Since the plasma gases are discarded to ambient atmosphere, the use of reactive gases such as ammonia, methane, or nitrous oxide is not permissible [11]. Instead, noble gases such as helium and argon were used to generate plasma jets. Since it is easier to reach plasma conditions with noble gases than air, the power is typically lower. The reactivity of the plasma jet was also increased by the addition of small amounts of oxygen.
The possible applications of the plasma jets are not limited to cosmetics of hair fibers. A dry cleaning method can also prove useful for preparing specimen (hair, nail, scab, etc.) for analytical analysis. The ease or resistance to surface modification upon plasma treatment may also be employed as a forensics and/or diagnostic tool.

\section{Experimental}

Hair samples were taken from two Caucasian female donors in early $20 \mathrm{~s}$. One donor's hair has never been dyed, i.e., virgin hair, and the other sample had dye on it. Single strands of hair about $50 \mathrm{~cm}$ were taken; the first and last $5-\mathrm{cm}$ segments were discarded. The remaining part was cut into 5-7-cm-long pieces and suspended across boat-shaped homemade ultra-high vacuum (UHV) compatible holders for plasma treatment. The length of hair fiber available for treatment was about $3 \mathrm{~cm}$. The center part of the hair segment was treated with atmospheric pressure helium, helium/oxygen, argon, and argon/oxygen plasma jets for $10 \mathrm{~min}$.

For comparison, hair samples were also treated for 10 min with mild hydrogen peroxide solution $(3 \%$ w) basified with ammonia (100:1 v/v). The 5-7-cm-long fiber strips were placed in the freshly prepared solutions. Gas evolution near fiber surface was observed during the treatment indicating the degradation of hydrogen peroxide. At the end of treatment time, the fibers were rinsed with deionized water, dried under ambient conditions, and placed on the UHV compatible holder.

The homemade atmospheric pressure cold plasma system is based on dielectric breakdown design with a central electrode in a cylindrical glass tube (6-mm diameter) and a ring outer electrode. The excitation is provided by a 1:800 transformer energized by a DC power supply and triggered with a MOSFET at $1-10 \mathrm{kHz}$. The $V_{\mathrm{pp}}$ is about $12 \mathrm{kV}$, and the average power drained from the DC supply is about $20 \mathrm{~W}$. Cold plasmas are generated by $\mathrm{He}, \mathrm{He}-1 \% \mathrm{O}_{2}$, Ar, and Ar- $1 \% \mathrm{O}_{2}$ gases, (mixtures) where the flow rates were adjusted with manual flow meters. The triggering frequency, flow rate, and duty cycle were optimized for (near) resonance operation. The plasma jet diameter was about $3 \mathrm{~mm}$ and covered the entire diameter of the fiber. The distance between the torch and the hair fiber was about $10-15 \mathrm{~mm}$. Plasma treatment was carried out by placing a single hair fiber, stretched across the UHV compatible holder, directly under the plasma jet under ambient conditions. The holder was placed in the experiment chamber within $1 \mathrm{~h}$ of the treatment.

The X-ray photoelectron (XPS) spectra were recorded with Thermo Scientific K-alpha model XPS using monochromatized $\mathrm{Al} \mathrm{K}_{\alpha}$ radiation $(1486.7 \mathrm{eV})$ with a spot size 
of 300 micrometer on the single hair fiber. The fiber diameter was smaller than the X-ray spot size; however, since the fiber was suspended, superfluous background signal from the sample holder was not generated. A lowenergy flood gun $\left(\mathrm{e}^{\prime}, \mathrm{Ar}^{+}\right)$was used for charge compensation. The pass energy of $50 \mathrm{eV}$ corresponds to an energy resolution of roughly $0.5 \mathrm{eV}$. The base pressure of $1 \times 10^{-9}$ mbar rose to $1 \times 10^{-7}$ mbar under operation. The spectra are referenced for the $\mathrm{C} 1 \mathrm{~s}$ signal at $285.0 \mathrm{eV}$ and intensity normalized for each hair sample with respect to the C $1 \mathrm{~s}$ spectra. The Avantage v5 software by Thermo Fischer Scientific was used for quantification.

The FE-SEM analysis was conducted with a Zeiss Ultra Plus field-emission scanning electron microscope operating at low voltage $(0.5-2 \mathrm{kV})$ to avoid surface damage and charging. Additional conductive coating was not applied to the samples.

The plasma treatments were repeated three times. The XPS spectra, for each sample, were collected from 3-5 different points within the region exposed to the plasma jet. The quantitative results were reproducible within $\pm 5 \%$. The representative images and spectra are presented here.

\section{Results}

\subsection{Virgin Hair}

The FE-SEM images of virgin hair, virgin hair treated with atmospheric pressure helium, helium/oxygen, argon, and argon/oxygen plasma jets for $10 \mathrm{~min}$ are presented in Fig. 1. For comparison, virgin hair treated with basic hydrogen peroxide solution is also presented. The virgin hair (Fig. 1a) has the overlapping cuticle structure with organic residues on and in between the layers of the cuticle. The organic residues are most likely 18-MEA and any other matter gathered from the environment (scalp) and hair care products.

Treatment of virgin hair with helium plasma jet for $10 \mathrm{~min}$ (Fig. 1b) removed the residues on the cuticles. After the treatment, only the residues in between the layers remained since the plasma jet could not reach these locations. The surface morphology of fiber remained unchanged. Introduction of oxygen into the helium plasma (Fig. 1c) not only cleaned the surface but also created a slightly rough surface covering the entire epicuticle. This new formation is most likely due to the oxidation of the organic/inorganic matter on the surface. The argon plasma jet treatment of the virgin hair surface (Fig. 1d) leaved a clean surface without organic residues; however, there was minor surface damage in terms of discoloration and pitting. The lack of organic coating on the surface is also deduced from the quality of the SEM image recorded under the same conditions as others. Argon plasma seems to have a greater cleaning effect than helium; even the spaces between the layers were cleaned and crisp SEM images could be taken. Mixing oxygen with argon (Fig. 1e) removed the residual organics effectively and high-quality SEM images could be taken. However, it also generated small humps all over the surface causing increased roughness. This effect is much more significant compared to the $\mathrm{He} / \mathrm{O}_{2}$ plasma jet case. The virgin hair sample treated with basic hydrogen peroxide (Fig. 1f) on the other hand did not show signs of severe cleaning or etching on the surface. The residual matter on and in between the layers was still highly visible.

The detailed XPS analysis of plasma jet-treated virgin hair is presented in Fig. 2. The bottom spectra belong to untreated virgin hair. The surface carbon content is about $80 \%$ mostly due to the aliphatic carbon from 18-MEA registered at a binding energy of $285.0 \mathrm{eV}$. A weak shoulder on the higher binding energy side in the $\mathrm{C} 1 \mathrm{~s}$ region reveals the presence of carbonyl and carboxyl species. These species, and the nitrogen-, oxygen-, and sulfurcontaining moieties, are due to the protein layers under the 18-MEA layer. This is a valid assumption since XPS is sensitive to the top $3-5 \mathrm{~nm}$ of the surface due to the inelastic electron mean free path [29], and the length of a $\mathrm{C} 18$ carbon chain is about $2.1 \mathrm{~nm}$ [30]. The symmetric $\mathrm{N}$ $1 \mathrm{~s}$ peak, centered at $400.3 \mathrm{eV}$, is indicating the presence of amine groups due to the amino acids present in the epicuticle layer [29]. The nitrogen content is about $3.5 \%$. The $\mathrm{O} 1 \mathrm{~s}$ peak is positioned at $532.4 \mathrm{eV}$ for carboxyl moiety of the peptides with an abundance of $13.3 \%$. The $\mathrm{S} 2 \mathrm{p}$ region shows the presence of two different sulfur moieties; the organic sulfur, i.e., $\mathrm{S}-\mathrm{C}$ or $\mathrm{S}-\mathrm{S}$, at $163.9 \mathrm{eV}$ from the cystine groups and an oxidized sulfur moiety at $168.7 \mathrm{eV}$ [29]. The sulfur peak at $168.7 \mathrm{eV}$ is assigned to the cysteic acid $\left(-\mathrm{SO}_{3}{ }^{-}\right)$group, which arises due to the stripping of the 18-MEA layer [19]. This reveals that the virgin hair fiber has regions where the 18-MEA layer is already stripped. The intensity of the oxidized sulfur moiety is higher than the organic sulfur groups. However, the unexpected intensity pattern can be explained by the attenuation of the photoelectrons from the organic sulfur moieties by the long carbon chain lipid layer, since the virgin hair is expected to have extensive 18-MEA coverage. The amount of total surface sulfur is low, ca. $0.8 \%$, which yields a non-stoichiometric $\mathrm{C} / \mathrm{S}$ ratio for pure 18-MEA layer. This observation also supports the fact that the observed XPS spectra have contributions from the underlying protein layer. Finally, Si $2 p$ region reveals the presence of $2.3 \%$ silicon due to the use of hair care products. The binding energy of $102.3 \mathrm{eV}$ confirms the siloxane structure [29].

Figure 2 also shows the XPS results of virgin hair treated with helium, helium/oxygen, argon, and argon/ 
Fig. 1 FE-SEM images of a virgin hair, virgin hair treated with b $\mathrm{He}$ plasma, c $\mathrm{He} / \mathrm{O}_{2}$ plasma, d Ar plasma, e $\mathrm{Ar} / \mathrm{O}_{2}$ plasma, $\mathbf{f ~} \mathrm{H}_{2} \mathrm{O}_{2}$ solution
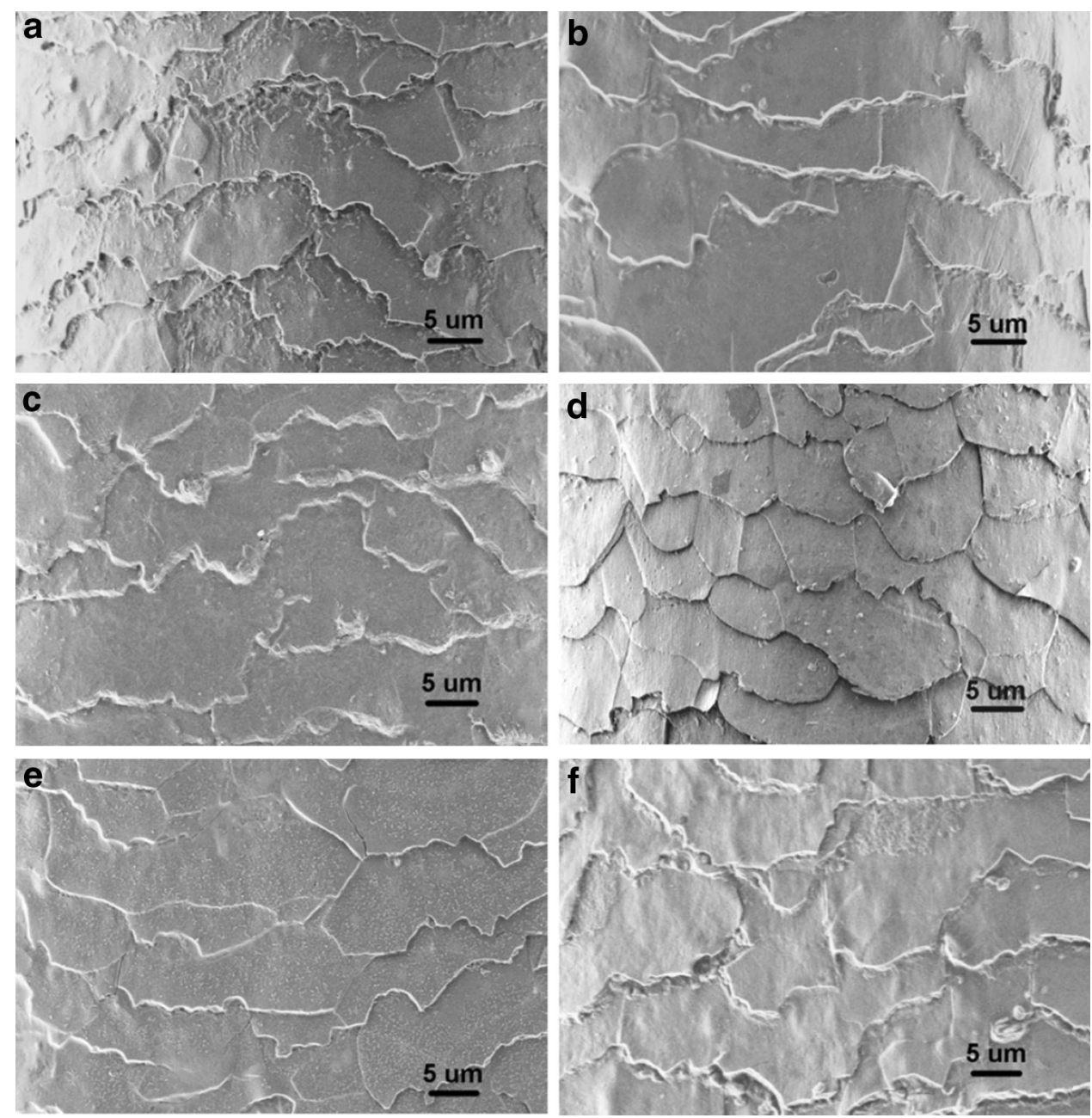

oxygen plasma jets for $10 \mathrm{~min}$ as well as hair treated in basic hydrogen peroxide for $10 \mathrm{~min}$. For the hair treated with helium plasma jet, the clear increase in the intensity of the high-binding energy side of $\mathrm{C} 1 \mathrm{~s}$ reveals the oxidation of surface carbon species. At the same time, the carbon amount decreased to $60.5 \%$ which is consistent with the SEM observations. The increase in the oxidized carbon species was also accompanied with the increase in the oxygen content of the surface from 13.3 to $26.2 \%$. This increase is most likely due to the reaction between oxygen and/or water vapor with the radical groups on the surface generated during the plasma jet treatment. At the same time, the nitrogen amount increased slightly to $6.6 \%$ and oxidized nitrogen species arose in the higher binding energy side of the $\mathrm{N} 1 \mathrm{~s}$ spectrum. A significant change in the $S 2 p$ spectrum was observed; the oxidized sulfur amount increased several folds and the total sulfur content increased to $2.7 \%$. The silicon $2 \mathrm{p}$ peak showed a slight shift in the binding energy to $103.2 \mathrm{eV}$ indicating the partial oxidation of the silicon species. The amount of silicon also increased to $4.0 \%$. One can argue that upon removal of the organic residue, nitrogen, sulfur, and silicon species are more exposed on the surface and slight increases in the quantities were detected. For these species, the oxidation of moieties is also evident due to the increasing intensity of peaks on the high binding energy side. However, alternative explanations are also possible for the increased quantities. It is well known that nitrogen in air can be excited and ionized by the metastable helium atoms present in the helium plasma jet [31, 32]. Therefore, nitrogen addition to the organic structure is also a possibility. The increase in the oxidized sulfur moiety signifies that the helium plasma jet application strips the 18-MEA layer, and the cysteic acid groups are now exposed on the top surface.

Introduction of $1 \%$ oxygen into the helium plasma modified the surface similar to the helium plasma jet case. The $\mathrm{C} 1 \mathrm{~s}$ region showed a small increase in the high binding energy side, while the total carbon amount decreased to $61.4 \%$. The $\mathrm{N} 1 \mathrm{~s}$ peak broadened toward the higher binding energy side, the $\mathrm{O} 1 \mathrm{~s}$ peak slightly shifted to higher binding energy, the intensity of the $S 2 p$ peak for 
Fig. 2 XPS spectra of virgin hair and hair treated with $\mathrm{He}$, $\mathrm{He} / \mathrm{O}_{2}, \mathrm{Ar}, \mathrm{Ar} / \mathrm{O}_{2}$ plasma jets and $\mathrm{H}_{2} \mathrm{O}_{2}$

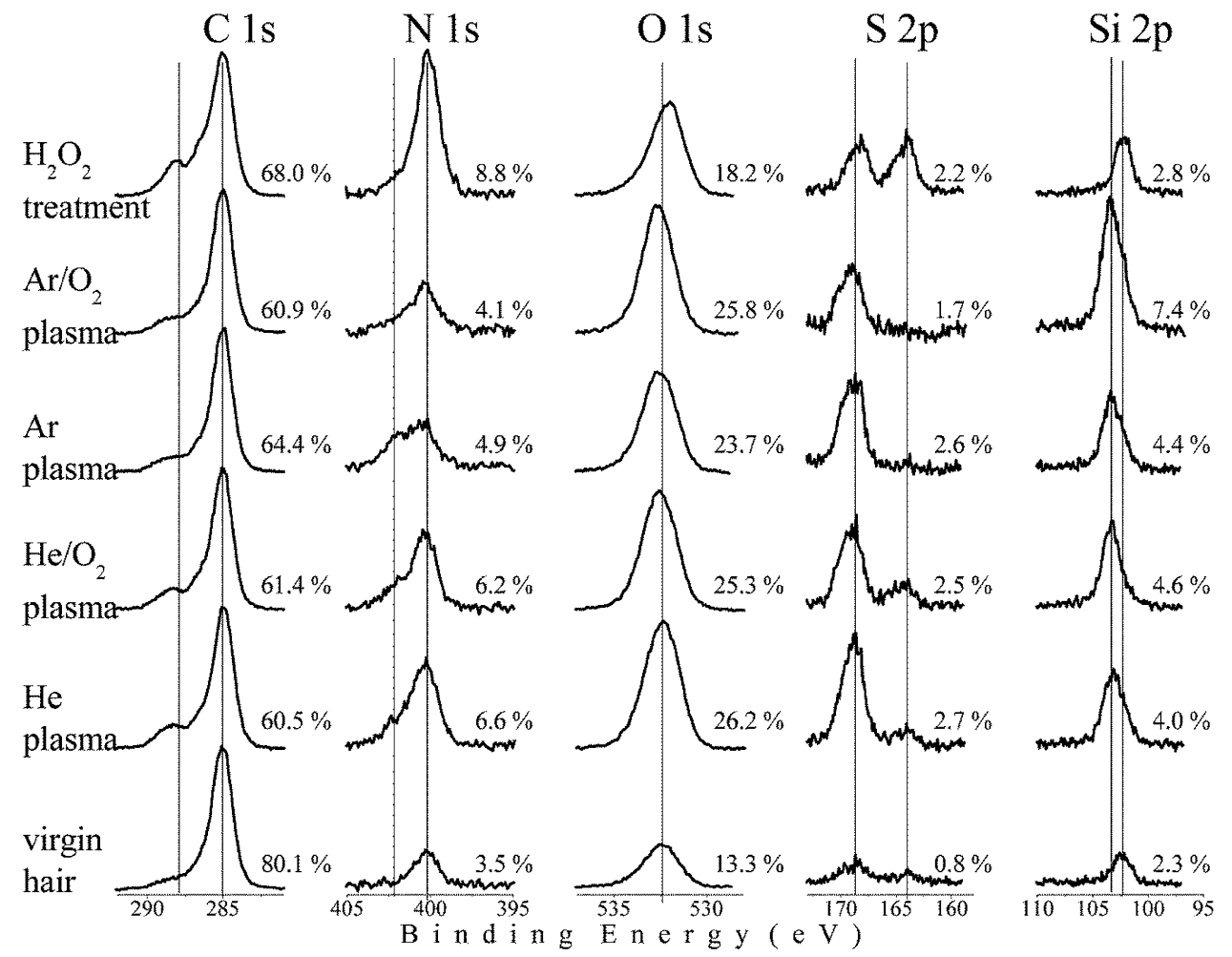

cysteic acid groups increased, and the Si $2 p$ peak shifted toward higher binding energy side. Comparable to the helium plasma jet case, the nitrogen, sulfur, and silicon species previously located at the subsurface moved toward the top surface upon the removal of the residual organic layer.

Argon plasma jet treatment of virgin hair was quite effective in cleaning the surface hydrocarbons. Although the amount of carbon $(64.4 \%)$ is comparable to previous cases, the SEM images corroborate to the better cleaning performance of argon. The reason of similar C $1 \mathrm{~s}$ intensity is the underlying organic content, i.e., cuticle, which is common for all hair samples. In addition to the better cleaning performance, the argon seems to have higher oxidation efficiency as deduced by the nitrogen and oxygen $1 \mathrm{~s}$ signals. The oxidized nitrogen species at $402.2 \mathrm{eV}$ gained significant intensity with argon plasma jet treatment compared to other treatments. The increase in the nitrogen content is not as much as the previous cases, i.e., $4.9 \%$; however, this may be an indication of a mild etching process, as well. The binding energy shift of the $\mathrm{O} 1 \mathrm{~s}$ peak to $532.7 \mathrm{eV}$ also supports the higher oxidation efficiency of the argon plasma jet. The oxygen content at $23.7 \%$ is comparable to previous cases. The $\mathrm{S} 2 \mathrm{p}$ region was dominated by the cysteic acid groups at $168.6 \mathrm{eV}$ with $2.6 \%$ abundance. The organic sulfur content around $164 \mathrm{eV}$ is quite weak indicating a near-complete stripping of the 18-MEA layer. This is probably the reason of much crispier
SEM image since charging of surface is less likely to happen when it is almost completely covered with anionic cysteic acid groups. The $\mathrm{Si} 2 \mathrm{p}$ region showed the presence of $\mathrm{Si}^{4+}$ species at $103.3 \mathrm{eV}$ as well as less oxidized moieties with an overall abundance of $4.4 \%$.

Introduction of $1 \%$ oxygen to argon plasma jet altered the surface composition slightly compared to argon only jet, despite the morphological changes observed in SEM images. The carbon content decreased to one of the lowest values observed, i.e., $60.9 \%$, with contributions from oxidized carbon species. The nitrogen signal was a little lower than the argon only jet, i.e., $4.1 \%$, with significantly less contribution of the oxidized nitrogen species. The $\mathrm{O}$ $1 \mathrm{~s}$ peak was shifted to slightly higher binding energies and reached the maximum amount, i.e., $25.8 \%$ among all the samples investigated. The sulfur species were almost completely the cysteic acid moieties at $167.7 \mathrm{eV}$; however, the amount, $1.7 \%$, was the lowest among all the plasma jet treatment cases. The silicon amount also presented another extreme; the quantity of $7.4 \%$ was the highest among all samples. The majority of silicon species had +4 oxidation state with binding energy at $103.3 \mathrm{eV}$ in addition to the less oxidized moieties at lower binding energy values. These results demonstrate the corrosive nature of the argon/oxygen plasma jet. The argon/oxygen jet treatment clearly has started to remove the cysteic acid groups from the surface causing a decrease in the sulfur amount. The increase in the silicon amount is related to the small humps observed with 
SEM covering the entire surface. The oxidized silicon species $\left(\mathrm{SiO}_{2}\right)$ bind to the surface and enrich while the organic material is removed, probably in the form of volatile oxides, during the mild etching process.

For comparison, the virgin hair was also treated with $3 \% \mathrm{H}_{2} \mathrm{O}_{2}$ solution as described in the experimental part. Peroxide treatment decreased the carbon amount from 80.1 to $68.0 \%$ while generating significant amounts of oxidized carbon species. The nitrogen amount increased significantly to $8.8 \%$ due to the presence of ammonia in the solution. The $\mathrm{O} 1 \mathrm{~s}$ peak, contrary to other cases, was positioned at $531.5 \mathrm{eV}$ and its amount was registered as $18.2 \%$. The opposite shift in the oxygen position signifies the differences in the chemical/physico-chemical processes involved in removing the 18-MEA layer. This effect is also visible in the unshifted binding energy position of the silicon species. The sulfur $2 p$ region showed the presence of two different sulfur species, organic sulfur at $163.5 \mathrm{eV}$, and the cysteic acid moieties at $167.7 \mathrm{eV}$ with comparable intensity. The total amount of surface sulfur was greater than that of virgin hair. Contrary to the deduction from the SEM images, the $\mathrm{H}_{2} \mathrm{O}_{2}$ treatment did generate chemical changes on the surface by removing the 18-MEA layer partially. However, the extent of this chemical change was not as significant as plasma jet treatment, nor it shared the same chemical processes.

\subsection{Dyed Hair}

The FE-SEM images of the dyed hair, dyed hair treated with helium, helium/oxygen, argon, and argon/oxygen plasma jets and $\mathrm{H}_{2} \mathrm{O}_{2}$ solution are presented in Fig. 3. The dyed hair surface looks quite different than the virgin hair (Fig. 3a). A thick layer coats the epicuticle layer and fills the gaps between the layers. Residual organic matter, most likely from the scalp lipids, is visible on the step edges of the cuticle layers and on the sides of the image. Helium and argon plasma jet treatments were successful in removing the organic residue, but they did not induce any noticeable morphology changes on the surface (Figs. $3 b$ and d, respectively). Introduction of oxygen into the helium plasma jet not only cleaned the organic matter, but also generated pitting corrosion on the coating (dye) layer as indicated with arrows in Fig. 3c. Argon/oxygen plasma jet treatment (Fig. 3e), on the other hand, lead to an overall coverage of the surface with small lumps, most likely oxidation products. These lumps increased the surface roughness of the fiber significantly. Hydrogen peroxide treatment of dyed hair (Fig. 3f) did not induce noticeable morphology change within $10 \mathrm{~min}$.

The XPS analysis of the dyed hair in "as is", plasma jetand hydrogen peroxide-treated forms are presented in Fig. 4. The dyed "as is" hair has C $1 \mathrm{~s}$ peak at $285.0 \mathrm{eV}$ with a small shoulder on the high binding energy side indicating the small amount of carbonyl and carboxyl species on the surface. The surface carbon amount was $61.5 \%$. The $\mathrm{N} 1 \mathrm{~s}$ region showed the presence of $3.6 \%$ nitrogen in the form of amine and nitro groups at 400.1 and $402.8 \mathrm{eV}$, respectively. The surface oxygen amounted to $21.9 \%$ with a binding energy of $532.5 \mathrm{eV}$. The sulfur $2 \mathrm{p}$ region shows the presence of two different kinds: the organic sulfur at $164.0 \mathrm{eV}$ and the oxidized sulfur at $168.0 \mathrm{eV}$. The total amount of sulfur was $1.1 \%$ with $2 / 3$ of them in oxidized form. The amount of silicon was quite high due to the dye, $11.9 \%$, and the binding energy of $102.2 \mathrm{eV}$ indicated the presence of siloxane species.

Helium plasma jet treatment of the dyed hair fiber created minor changes on the surface chemistry. The $\mathrm{C} 1 \mathrm{~s}$ region seems to have lower intensity around $287 \mathrm{eV}$, i.e., carbonyl region, whereas the intensity around $289 \mathrm{eV}$, i.e., carboxyl region, increased. The oxidation of surface carbon was also accompanied by the increase in the oxygen amount to $25.0 \%$. The helium plasma jet treatment diminished the nitrogen and sulfur amounts and left the surface with the oxidized moieties of these elements. The silicon amount remained essentially the same; however, the oxidation of the silicon species with helium jet treatment was evident by the shift in the Si $2 p$ binding energy to $103.2 \mathrm{eV}$, i.e. $\mathrm{Si}^{4+}$ form. These chemical changes could be attributed to effective cleaning of the helium plasma jet with very mild surface erosion.

$\mathrm{He} / \mathrm{O}_{2}$ plasma jet treatment of the surface led to slightly less carbon amount $(57.8 \%)$, while the higher binding energy side of the $\mathrm{C} 1 \mathrm{~s}$ spectrum also slightly lost intensity. The nitrogen, sulfur, and silicon amounts were slightly higher than helium only plasma jet, and all shifted to higher binding energy values indicating the oxidation potential of the plasma jet. The slight decrease in the carbon amount and the slight increase of the other elements in the surface composition can be attributed to the light corrosive effect of the plasma jet. Since the SEM results also showed the minor pitting erosion, one can argue that these small changes in the elemental composition were due to exposure of the subsurface.

The argon plasma jet treatment had a discernable oxidation effect on the surface; it generated oxidized carbon, nitrogen, sulfur, and silicon species. The total carbon amount decreased to $53.2 \%$ while the carboxyl species increased. Nitrogen amount increased to $6.3 \%$ with the nitro groups $(402.5 \mathrm{eV})$ as intense as the amine groups $(400.1 \mathrm{eV})$. A similar trend was also generated with the sulfur content; the amount increased to $2.9 \%$ and was dominated by the oxidized sulfur species, i.e., cysteic acid moieties. The oxygen amount increased to $29.2 \%$ and the peak broadened in parallel to the multitude of the oxidized species on the surface. Due to the increase in other species, 
Fig. 3 FE-SEM images of a dyed hair, dyed hair treated with b $\mathrm{He}$ plasma, c $\mathrm{He} / \mathrm{O}_{2}$ plasma, d Ar plasma, e $\mathrm{Ar} / \mathrm{O}_{2}$ plasma, $\mathbf{f} \mathrm{H}_{2} \mathrm{O}_{2}$ solution
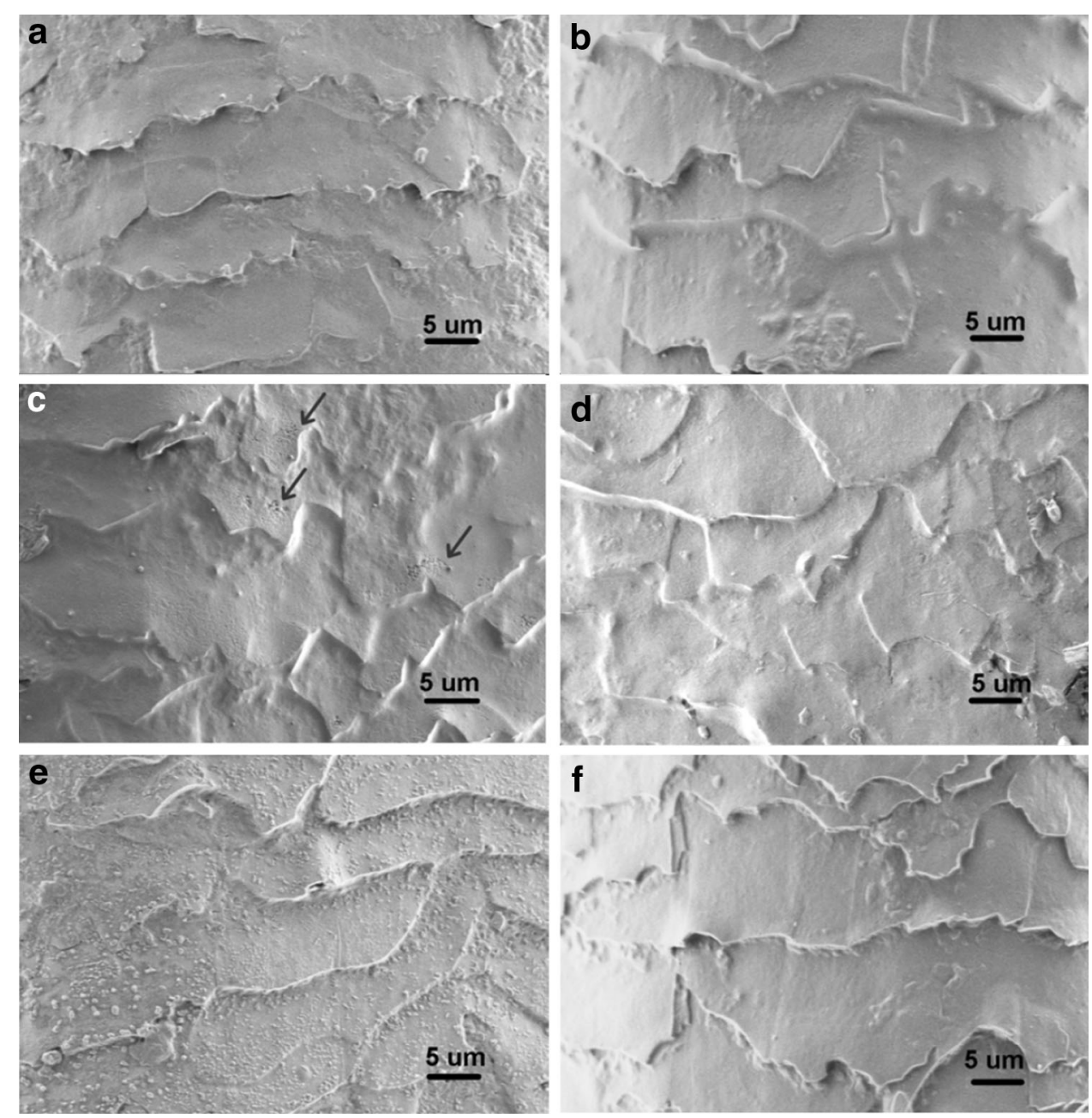

a decrease to $8.4 \%$ in the silicon amount was detected. The silicon oxidation state was determined as +4 . The increases in the nitrogen and sulfur species with respect to the "as is dyed fiber" stems from the slight corrosion effect of the argon jet plasma such that the subsurface species, i.e., protein cysteine moieties, can be exposed afterward.

Introduction of oxygen to the argon plasma on the other hand significantly altered the surface chemistry as well as the morphology. While the total carbon amount decreased to $41.0 \%$, a significant intensity was registered for the oxidized carbonyl and carboxyl species. The nitrogen content increased to $9.1 \%$ comparable intensity in the amine and nitro groups present. The oxygen amount shot up to $36.0 \%$ and the surface sulfur amount increased to the highest value of $3.6 \%$ in the oxidized form. The silicon was converted to $\mathrm{Si}^{4+}$ with a binding energy of $103.4 \mathrm{eV}$ and the amount was $10.3 \%$. The structures observed in the SEM image are most likely the silicon oxidation products bound to the surface. However, the enrichment effect was not as significant as the $\mathrm{He} / \mathrm{O}_{2}$ plasma jet case due to already high silicon amount and thick overcoat. $\mathrm{The} \mathrm{Ar} / \mathrm{O}_{2}$ plasma jet has a significant oxidation effect on the dyed hair as well as a moderate corrosive effect.

The hydrogen peroxide treatment of dyed hair resulted in the selective cleaning of the oxidized carbon species. The carbon amount remained essentially unchanged with respect to the "as is" dyed hair, while the amount of oxidized carbon decreased. Slight decreases in the nitrogen, oxygen, and sulfur amount were detected. The silicon amount increases slightly with respect to the "as is" dyed hair, which is due to the overall decrease in the other species. However, the oxidation state of silicon, as deduced from the unchanged binding energy, remained the same. Therefore, the hydrogen peroxide treatment on the dyed hair could not induce as significant chemical and morphological changes as the treatment of the virgin hair with the used formulation and duration.

\section{Discussions}

The epicuticle layer of human hair fibers is covered inhomogenously with a lipid layer (18-MEA) which is 
Fig. 4 XPS spectra of dyed hair and dyed hair treated with $\mathrm{He}$, $\mathrm{He} / \mathrm{O}_{2}, \mathrm{Ar}, \mathrm{Ar} / \mathrm{O}_{2}$ plasma jet and $\mathrm{H}_{2} \mathrm{O}_{2}$ solution

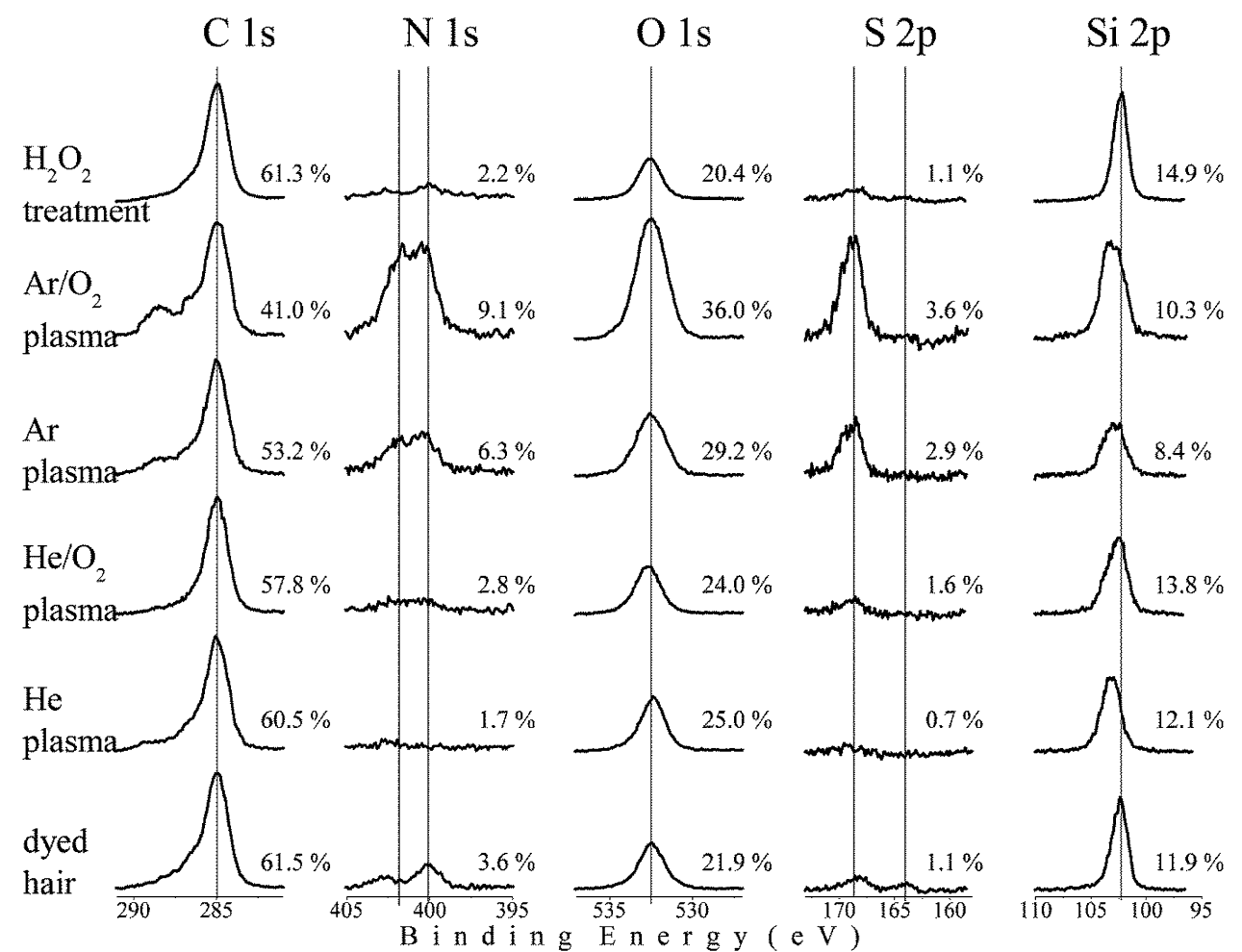

attached to the underlying protein layer via chemical linkers. The removal of the 18-MEA layer exposes the oxidized thioester linkers, i.e., cysteic acid $\left(-\mathrm{SO}_{3}{ }^{-}\right)$groups, hence Si 2p XPS spectra could be used to assess the coverage of the layer. The XPS spectrum of virgin hair showed that some of the 18-MEA layer was already removed from these fibers since the oxidized sulfur signal could already be detected. The plasma jet treatment clearly stripped off the lipid layer and exposed the subsurface. During the 10 min of plasma treatment, regardless of the gas type used, the carbon percentage decreased, oxygen percentage increased together with the increase in the oxidized species of nitrogen, sulfur, and silicon. The cleaning effect of the Ar plasma jet was more potent than that of Helium plasma jet, which could not completely clean the space between the platelet overlays. However, XPS could barely detect these differences since the remaining residues were out of direct sight of the incoming X-ray beam. The differences in the surface compositions must be interpreted together with changes in the surface morphology. Oxygen-containing plasma jets had a corrosive nature that created a rougher surface decorated with sub-micrometer scale bumps. These bumps are most probably $\mathrm{SiO}_{2}$ islands formed during oxidative plasma treatment. The argon/oxygen plasma jet was more corrosive than the helium/oxygen plasma jet, which is evident by the extent of surface damage created. Those samples also had lesser nitrogen and sulfur remaining on the surface due to etching.
The applied hydrogen peroxide treatment was not as successful as the plasma treatment in cleaning the organic residues off the surface. Although a decrease in the carbon amount could be detected, the presence of organic sulfur and unaltered position of the silicon peak lead to the conclusion that the oxidation potential of the plasma treatment was significantly higher and the kinetics was faster. The hydrogen peroxide degradation creates the highly reactive hydroxyl radical, which reacts with surface moieties and degrade them into fragments. This reaction seems to be effective in oxidizing carbon species, however not so much toward nitrogen, sulfur, and silicon. The plasma jet, on the other hand, provides several different competing reaction pathways including highly reactive radicals, metastable helium atoms, and ultraviolet radiation, all of which can attack organic matter as well as silicon. For this reason, all of the plasma jet versions seem to effectively oxidize all the surface species. The differences between the helium and argon plasmas probably stem from the differences in their ionization energies (24.6 vs. $15.8 \mathrm{eV}$ ) and the densities of radicals, ions, and metastable atoms in the plasma jets.

The dye coating on the hair was so thick that a 10-min atmospheric pressure plasma jet treatment could not etch it completely. The helium plasma jet had a mild cleaning effect, while argon plasma jet had a mild corrosive effect on the surface. Introduction of oxygen into the plasma increased the corrosive characters relatively. The argon/ 
oxygen plasma jet was much more corrosive than the helium/oxygen plasma jet. The argon/oxygen plasma jet effectively stripped off the layers of dye and exposed the underlying layers. For the same treatment time, argon/ oxygen plasma jet application proved to be a better etcher than mild basic hydrogen peroxide.

This work unveils the potential of the atmospheric pressure plasma jets to replace the classical hydrogen peroxide treatment. The chemistry leading to the removal of the lipid layer is different than the peroxide treatment case. A helium or argon plasma jet is highly capable of cleaning the residual organic matter from the hair fiber surface, exposing the cysteic acid groups and generating oxidized carbon and nitrogen groups which can bind to the polar dye groups effectively. The effect is limited to the top few nanometers of the fiber surface; therefore, bulk damage to the fiber is avoided. The plasma treatment also has the advantage of acting on all surface species without particular chemical selectivity.

The oxygen-containing plasma jets have more corrosive character and can be used to treat more resistant types of hairs or coatings. The corrosive behavior of these types of plasma jets was observed both for the virgin and dyed hair. While it is not probably healthy to apply to untreated hair, their potential for restoring hair to original condition or color should be explored.

Furthermore, noble gas atmospheric pressure plasma jet treatment proved to be a fast method to clean hair fibers before analytical analyses. There are several techniques such as electron microscopy, optical microscopy, color testing, light scattering, elemental analysis, laser ablation, etc. which may benefit from the removal of organic residues from the surface of the hair. Pretreatment of hair by atmospheric pressure plasma jets may improve the quality and reproducibility of such investigations. Finally, the study must be extended to include age, gender, race, and type differences to investigate the potential for differentiation toward diagnostic or forensic applications.

\section{Conclusions}

Human hair fibers in virgin and dyed form were treated with atmospheric pressure plasma jets run with different gases at low power. The changes generated on the surface of the hair were studied by electron microscopy and X-ray photoelectron spectroscopy. Ten-minute noble gas plasma jet treatment of virgin hair was sufficient to clean organic contaminants and create oxidized functional groups. The mild hydrogen peroxide treatment with the same duration could not reach the same performance. Therefore, these types of plasma jets have the potential to replace peroxide treatment. The plasma jets can also gain corrosive properties when oxygen is added to the gas mixture. These types of corrosive jets can also be used to restore dyed hair fibers. In addition, the jets can be used to clean the surfaces of hair fibers to prepare samples for analytical investigations where the organic residues may create interference or hardship.

Acknowledgments This work was supported by Istanbul Rotary Club. On behalf of all authors, the corresponding author states that there is no conflict of interest.

\section{References}

1. Bárdos L, Baránková H (2010) Cold atmospheric plasma: sources, processes, and applications. Thin Solid Films 518(23):6705-6713. doi:10.1016/j.tsf.2010.07.044

2. Nehra V, Kumar A, Dwivedi H (2008) Atmospheric non-thermal plasma sources. Int J Eng 2(1):53-68

3. Pappas D (2011) Status and potential of atmospheric plasma processing of materials. J Vac Sci Technol A 29 (2). doi:10.1116/ 1.3559547

4. Tendero C, Tixier C, Tristant P, Desmaison J, Leprince P (2006) Atmospheric pressure plasmas: a review. Spectrochim Acta Part B 61(1):2-30. doi:10.1016/j.sab.2005.10.003

5. Kogelschatz U (2004) Atmospheric-pressure plasma technology. Plasma Phys Control Fusion 46(12B):B63

6. Morent R, De Geyter N, Verschuren J, De Clerck K, Kiekens P, Leys C (2008) Non-thermal plasma treatment of textiles. Surf Coat Technol 202(14):3427-3449. doi:10.1016/j.surfcoat.2007. 12.027

7. Conrads H, Schmidt M (2000) Plasma generation and plasma sources. Plasma Sources Sci Technol 9(4):441

8. Jiang N, Ji A, Cao Z (2009) Atmospheric pressure plasma jet: Effect of electrode configuration, discharge behavior, and its formation mechanism. J Appl Phys 106 (1). doi:10.1063/1.3159884

9. Law VJ, Anghel SD (2012) Compact atmospheric pressure plasma self-resonant drive circuits. J Phys D Appl Phys 45(7):075202

10. Tsai PP, Wadsworth LC, Roth JR (1997) Surface modification of fabrics using a one-atmosphere glow discharge plasma to improve fabric wettability. Text Res J 67(5):359-369. doi:10. 1177/004051759706700509

11. Wakida T, Takeda K, Tanaka I, Takagishi T (1989) Free radicals in cellulose fibers treated with low temperature plasma. Text Res J 59(1):49-53. doi:10.1177/004051758905900107

12. Wakida T, Tokino S, Shouhua Niu, Kawamura H, Sato Y, Lee M, Uchiyama H, Inagaki H (1993) Surface characteristics of wool and poly (ethylene terephthalate) fabrics and film treated with low-temperature plasma under atmospheric pressure. Text Res J 63(8):433-438. doi:10.1177/004051759306300801

13. Wang CX, Liu Y, Xu HL, Ren Y, Qiu YP (2008) Influence of atmospheric pressure plasma treatment time on penetration depth of surface modification into fabric. Appl Surf Sci 254(8):2499-2505. doi:10.1016/j.apsusc.2007.09.074

14. Wang CX, Qiu YP (2007) Two sided modification of wool fabrics by atmospheric pressure plasma jet: Influence of processing parameters on plasma penetration. Surf Coat Technol 201(14):6273-6277. doi:10.1016/j.surfcoat.2006.11.028

15. Wang CX, Ren Y, Qiu YP (2007) Penetration depth of atmospheric pressure plasma surface modification into multiple layers of polyester fabrics. Surf Coat Technol 202(1):77-83. doi:10. 1016/j.surfcoat.2007.04.077 
16. Liu Z, Jones FR, Liu Y, Jiang B (2014) Adhesion of aqueous polyurethane adhesive to human hair. Int $\mathrm{J}$ Adhes Adhes 48:14-19. doi:10.1016/j.ijadhadh.2013.09.004

17. Bhushan B (2010) Biophys Human Hair. Springer, Berlin

18. Okamoto M, Tanji N, Habe T, Inoue S, Tokunaga S, Tanamachi H (2011) ToF-SIMS characterization of the lipid layer on the hair surface. II : effect of the 18-MEA lipid layer on surface hydrophobicity. Surf Interface Anal 43(1-2):298-301. doi:10.1002/sia. 3406

19. Robbins CR (2002) Chemical and physical behavior of human hair. Springer, Berlin

20. Negri AP, Cornell HJ, Rivett DE (1993) A model for the surface of keratin fibers. Text Res J 63(2):109-115. doi:10.1177/ 004051759306300207

21. Breakspear S, Smith JR, Luengo G (2005) Effect of the covalently linked fatty acid 18-MEA on the nanotribology of hair's outermost surface. J Struct Biol 149(3):235-242. doi:10.1016/j. jsb.2004.10.003

22. Baghdadli N, Luengo GS, Recherche L (2008) A closer look at the complex hydrophilic/hydrophobic interactions forces at the human hair surface. J Phys: Conf Ser 100(5):052034

23. Wortmann FJ, Wortmann G, Schulze zur Wiesche E (2010) Spatial Probing of the Properties of the Human Hair Surface Using Wilhelmy Force Profiles. Langmuir 26(10):7365-7369. doi:10.1021/la9043842

24. Horr TJ (1997) A description of the wool fiber surface based on contact angle measurements. Text Res J 67(1):1-5. doi:10.1177/ 004051759706700102
25. Korte M, Akari S, Kühn H, Baghdadli N, Möhwald H, Luengo GS (2014) Distribution and localization of hydrophobic and ionic chemical groups at the surface of bleached human hair fibers. Langmuir 30(41):12124-12129. doi:10.1021/la500461y

26. Trüeb RM, Tobin DJ (2010) Aging Hair. Springer, Berlin

27. Boullion C, Wilkinson J (2005) The science of hair care. Taylor and Francis, Boca Raton

28. Tanamachi H, Tokunaga S, Tanji N, Oguri M, Inoue S (2010) 18-MEA and hair appearance. Int J Cosmet Sci 32(5):396-396. doi:10.1111/j.1468-2494.2010.00609_6.x

29. Briggs D, Seah P (1990) Practical surface analysis: Auger and X-ray photoelectron spectroscopy. Wiley, New York

30. Gupta RK, Suresh KA, Guo R, Kumar S (2006) LangmuirBlodgett films of octadecanethiol-properties and potential applications. Anal Chim Acta 568(1-2):109-118. doi:10.1016/j. aca.2005.10.010

31. Richardson WC, Setser DW (1973) Penning ionization optical spectroscopy: Metastable helium (He $23 \mathrm{~S}$ ) atoms with nitrogen, carbon monoxide, oxygen, hydrogen chloride, hydrogen bromide, and chlorine. J Chem Phys 58(5):1809-1825. doi:10.1063/1. 1679437

32. Ueno T, Hatano Y (1976) Measurement of the rate constant of the penning ionization of nitrogen by helium metastable states as studied by pulse radiolysis. Chem Phys Lett 40(2):283-286. doi:10.1016/0009-2614(76)85079-8 